\title{
Abordagem laboratorial das síndromes antifosfolípide e do aborto recorrente
}

\section{Laboratories analyses indicated in antiphospholipid and recurrent abortion syndrome}

\author{
Juliane Cristina Trevisan Sanches', Luciana Zambeli Caputto², Alexandre Luiz Affonso Fonseca', Enny Fernandes Silva³,
} Fernando Luiz Affonso Fonseca ${ }^{4}$

\section{Resumo}

Um aumento no potencial hemostático endometrial ocorre durante o ciclo menstrual, possibilitando, assim, a implantação embrionária, caso ocorra a fertilização. Defeitos nas proteínas de coagulação podem alterar esse estado de hipercoagulabilidade gestacional e desencadear perdas fetais por falhas na implantação ou na nutrição do embrião. A síndrome do aborto recorrente pode ser ocasionada por inúmeros fatores, entre eles anormalidades cromossômicas, anatômicas ou hormonais, ou ainda por defeitos nas proteínas de coagulação sanguínea ou plaquetária. Uma causa comum de abortos recorrentes é a síndrome antifosfolípide, uma desordem sistêmica, autoimune, caracterizada por trombose arterial e/ou venosa, morte fetal, abortos recorrentes e trombocitopenia, acompanhada de títulos elevados de anticorpos antifosfolípides: anticoagulante lúpico e/ou anticardiolipina. Pela íntima associação dessas síndromes, faz-se necessário investigá-las em pacientes que procuram os tratamentos de fertilização in vitro.

Palavras-chave: Síndrome antifosfolipídica; aborto habitual; inibidor de coagulação do lúpus; anticorpos anticardiolipina.

\begin{abstract}
An increase in endometrial hemostatic potential occurs during the menstrual cycle, thus enabling the embryonic implantation in the occurrence of fertilization. Defects in the coagulation proteins may change this state of gestational hypercoagulability and cause fetal losses by failures in the implantation or nutrition of the embryo. The recurrent abortion syndrome can occur by many factors, including chromosomal, anatomical or hormonal abnormalities or defects in proteins of blood or platelet coagulation. A common cause of recurrent miscarriages is antiphospholipid syndrome, a systemic disorder, autoimmune, characterized by arterial and/or venous thrombosis, fetal death, recurrent miscarriages, and thrombocytopenia, accompanied by evidence of elevated antiphospholipid antibodies: lupus anticoagulant and/or anticardiolipin. Due to the intimate association of these syndromes, the investigation of them is necessary in patients seeking for in vitro fertilization treatment.
\end{abstract}

Keywords: Antiphospholipid syndrome; abortion, habitual; lupus coagulation inhibitor; antibodies, anticardiolipin.

Recebido: $13 / 3 / 2009$

Revisado: $15 / 9 / 2009$

Aprovado: 17/10/2009

\footnotetext{
Curso de Especialização em Análises Clínicas do Instituto de Pesquisa e Educação em Saúde de São Paulo (IPESSP), São Paulo (SP), Brasil

2 Laboratório Chromos de Citogenética, São Paulo (SP), Brasil

3 Disciplina de Bioquímica da Faculdade de Farmácia e Bioquímica da Universidade Paulista (Unip), São Paulo (SP), Brasil

${ }^{4}$ Laboratório de Análises Clínicas da Disciplina de Hematologia e Oncologia da Faculdade de Medicina do ABC (FMABC), Santo André (SP), Brasil

Endereço para correspondência: Fernando Luiz Affonso Fonseca - Laboratório de Análises Clínicas da FMABC, Anexo 3 - Avenida Lauro Gomes, 2.000 -

CEP 09060-870 - Santo André (SP) - E-mail: laboratoriofmabc@yahoo.com.br
} 


\section{Introdução}

\section{Hemostasia endometrial}

A falha na implantação embrionária é considerada uma causa relevante de insucessos nos procedimentos de fertilização in vitro (FIV) ${ }^{1}$. As possíveis causas de falha na implantação embrionária têm sido investigadas, mas não há consenso na literatura. Acredita-se que a qualidade embrionária e a receptividade endometrial constituam fatores relevantes no insucesso da FIV. Sugere-se que alterações sanguíneas que levam à hipercoagulabilidade, ou seja, as trombofilias, possam comprometer o processo de implantação embrionária ${ }^{2}$.

O sucesso da gestação, placentação e subsequente gestação requerem desenvolvimento e remodelação vascular coordenados de ambos os lados da interface materno fetal. Um espessamento adequado da parede endometrial e um aumento na vascularização desta são necessários para a implantação embrionária. Tal remodelação da vasculatura endometrial é essencial para acomodar o rápido crescimento do embrião ${ }^{3}$.

Durante o ciclo menstrual, ocorre um aumento no potencial hemostático endometrial, essencial principalmente para a implantação embrionária. Durante a fase luteal do ciclo menstrual, a progesterona dá início ao processo de decidualização, e o estradiol estimula as células do estroma endometrial ao redor dos vasos sanguíneos a expressarem o fator tecidual (FT). O estímulo continuado da progesterona leva a uma extensa expressão de FT pelas células do estroma decidualizado por todo o endométrio na fase luteal e no endométrio gravídico ${ }^{4-7}$.

O FT, também denominado fator III da coagulação ou tromboplastina, é uma glicoproteína ligada à membrana que atua como um receptor para o fator VII (FVII) ${ }^{8,9}$. É expresso constitutivamente tanto nos sítios perivasculares, como na adventícia das artérias e veias, protegendo contra a injúria vascular. Por meio da ligação do FVII circulante no plasma ao FT ligado à membrana, forma-se o complexo TF-FVII e este, em presença de íons cálcio, promove

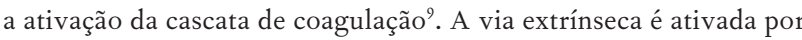
meio do fator X (FX) e a via intrínseca, pela ativação do fator IX $(\text { FIX) })^{8,10-12}$ (Figura 1).

A via extrínseca da coagulação tem um papel crucial na manutenção da hemostasia durante a gestação em humanos; isso é evidente após os eventos da implantação e subsequente placentação. O processo de invasão do trofoblasto inicia a circulação uteroplacentária primordial, que fornece ao embrião sua fonte vital de oxigênio e nutrientes enquanto remove dióxido de carbono e outros produtos de excreção. O citotrofoblasto atravessa as células deciduais, como trofoblastos extravilosos (EVT), que rompem e remodelam as artérias endometriais espiraladas ${ }^{13,14}$. Durante essa invasão do EVT, ocorre formação de fibrina por meio da expressão de FT pelas células deciduais, formando um "envelope hemostático", que protege contra hemorragia nos estágios inicias de invasão dos capilares e subsequente remodelação ${ }^{15}$.

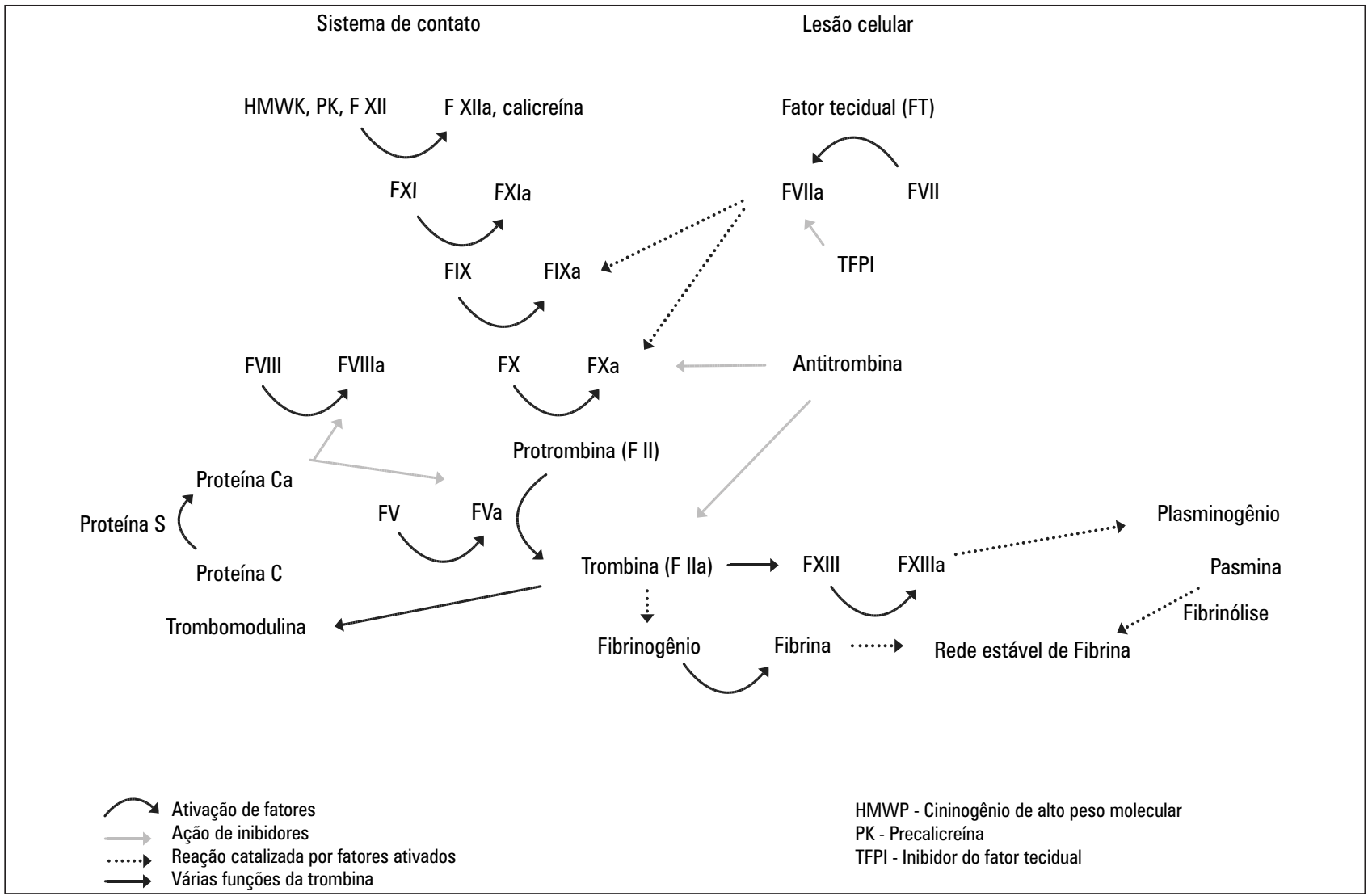

Figura 1 - Cascata de coagulação 
A circulação placentária é assegurada por modificações nas artérias espiraladas e pela hipercoagulabilidade gestacional. Desse modo, tem-se a elevação dos fatores pró-coagulantes e redução dos fatores anticoagulantes e da fibrinólise, induzindo estado de hipercoagulabilidade secundária $^{16}$. Defeitos nas proteínas de coagulação podem alterar esse estado de hipercoagulabilidade gestacional e desencadear perdas fetais por falhas na implantação ou na nutrição do embrião.

O FT é também essencial para outros processos celulares, incluindo a embriogênese e a angiogênese. A ausência do gene de FT resulta em alta mortalidade embrionária devido a combinação de defeitos hemostáticos e na vasculatura do saco vitelino ${ }^{15,17}$.

\section{Síndrome do aborto recorrente}

A síndrome do aborto recorrente pode ocorrer devido a anormalidades cromossômicas, anatômicas ou hormonais, ou ainda a defeitos nas proteínas de coagulação sanguínea ou plaquetária ${ }^{18}$. Os defeitos nas proteínas de coagulação ou plaquetárias podem ocorrer por dois mecanismos, associados a desordens hemorrágicas ou trombóticas ${ }^{19}$.

Os defeitos hemorrágicos associados à perda fetal são raros e decorrem da formação inadequada da fibrina, impossibilitando a implantação adequada. Podem estar relacionadas à deficiência dos fatores $\mathrm{XIII}^{20}, \mathrm{XII}^{21}, \mathrm{X}^{22}, \mathrm{VII}^{23}, \mathrm{~V}^{24}$, II (protrombina) ${ }^{25}$, síndrome de von Willebrand ${ }^{26}$ e portadores de hemofilia e de defeitos do fibrinogênio ${ }^{27}$.

Os defeitos trombóticos associados à perda fetal são mais comuns e decorrem da formação de trombos nos vasos placentários iniciais. Essas perdas ocorrem com maior frequência durante o primeiro trimestre, porém podem ocorrer no segundo ou terceiro ${ }^{19}$. A formação de trombos nos vasos placentários tanto arteriais como venosos impossibilitam a nutrição do embrião, inviabilizando, assim, seu desenvolvimento.

Entre os defeitos trombóticos associados às perdas fetais, podemos incluir: a presença do anticoagulante lúpico e dos anticorpos anticardiolipina, que compreendem a síndrome antifosfolípide ${ }^{28}$, deficiência do fator $\mathrm{XII}^{29}$, disfibrinogênias associadas com trombose ${ }^{30}$, defeitos nas proteínas $\mathrm{C} \mathrm{e} \mathrm{S}^{31}$, deficiência de antitrombina ${ }^{32}$, deficiência de heparina (cofator II) ${ }^{33}$, síndrome da agregação plaquetária, defeitos do fator $\mathrm{V}$ ou de Leiden e mutação da 5,10-metileno tetrahidrofolato redutase (5,10-MTHFR) e do gene G2010A da protrombina ${ }^{19}$. A síndrome antifosfolípide tem sido considerada causa comum da síndrome do aborto recorrente ${ }^{34}$.

\section{Síndrome antifosfolípide}

A síndrome antifosfolípide é uma desordem sistêmica, autoimune, caracterizada por trombose arterial e/ ou venosa, morte fetal, abortos recorrentes e trombocitopenia, acompanhada de títulos elevados de anticorpos antifosfolípides: anticoagulalante lúpico e/ou anticardioli- pina. Essa síndrome foi descrita originalmente em pacientes com lúpus eritematoso sistêmico (LES) $)^{35,36}$.

$\mathrm{O}$ anticorpo antifosfolípide, que reage contra antígenos de coração bovino, foi detectado em pacientes com sífilis, posteriormente identificado como cardiolipina, um fosfolípide mitocondrial ${ }^{37,38}$. Conley e Hartmann ${ }^{39}$ foram os primeiros a descrever a presença de anticoagulante circulante em pacientes com LES que, posteriormente, recebeu o nome de anticoagulante lúpico ${ }^{40}$.

Bowie et al. ${ }^{41}$ chamaram a atenção para a relação entre anticoagulantes sistêmicos em pacientes com LES e eventos trombóticos. Hughes $^{42}$ descreveu a associação entre anticorpos antifosfolípides e trombose arterial e venosa. A associação entre a presença desses anticoagulantes tem sido relacionada a abortos recorrentes há algum tempo, porém não está totalmente elucidada ${ }^{19,43}$.

A síndrome antifosfolípide pode ser classificada em primária ou secundária. A classificação secundária se dá quando esta é associada a doenças do colágeno, como lúpus eritematoso sistêmico, artrite reumatoide, esclerose sistêmica, entre outros, ou a infecções agudas ou crônicas (HIV, sífilis, malária), doenças linfoproliferativas etc?.

\section{Fisiopatologia}

Os mecanismos pelos quais os anticorpos antifosfolípides interagem com a cascata de coagulação ainda não foram bem elucidados. Sabe-se que a presença de lesão endotelial associada aos anticorpos antifosfolípides é requerida para uma complicação trombótica ${ }^{34}$.

Os mecanismos sugeridos para a indução dos anticorpos antifosfolípides a eventos trombóticos são:

1. interferência com os fosfolípides endoteliais e secreção de prostaciclinas;

2. inibição da precalicreína e inibição da fibrinólise;

3. inibição da trombomodulina e da atividade das proteínas C e S;

4. aquisição de resistência a proteína C;

5. interação com os fosfolípides da membrana plaquetária;

6. inibição da secreção de ativador de plasminogênio tecidual (tPA) endotelial;

7. inibição direta da proteína $S$;

8. inibição da anexina- $\mathrm{V}$, uma proteína de superfície celular que inibe o FT, também referida como "proteína anticoagulante placentária";

9. indução da liberação de FT pelos monócitos ${ }^{31}$.

\section{Diagnósticos}

Deve-se suspeitar de síndrome antifosfolípide quando ocorrem tromboses recorrentes ou quando existirem alterações gestacionais associadas a anormalidades laboratoriais específicas. O diagnóstico laboratorial deve incluir testes de coagulação para a pesquisa do anticoagulante lúpico e testes de fase sólida para o anticorpo anticardiolipina? (Quadro 1). 
Quadro 1 - Caracterização da síndrome antifosfolípide

\begin{tabular}{|l|}
\hline Critérios clínicos: \\
- trombose arterial ou venosa \\
decorrêencia de pré-eclampsia ou insuficiência placentária ou ainda por \\
abortamentos espontâneos) \\
\hline Critérios laboratoriais: \\
- presença do anticorpo anticardiolipina IgG ou IgM, com títulos eleva- \\
dos por meio da metodologia ELISA \\
presença do anticoagulante lúpico: \\
1. tempo de tromboplastina parcial ativado (TTPA), tempo do veneno \\
diluído da víbora de Russel (sRVVT) e tempo de coagulação com \\
caulim (KCT) \\
2. adição ao plasma teste de plasma normal na tentativa de se nor- \\
malizar o tempo de coagulação, evidenciando deficiência de fatores \\
da coagulação \\
3. adição de fosfolípides ao plasma teste, na tentativa de reduzir o \\
tempo de coagulação prolongado \\
\hline
\end{tabular}

A presença do anticorpo anticardiolipina é avaliada pelo método imunoenzimático (ELISA) ${ }^{43}$. Nesse método, a cardiolipina (fosfolípide aniônico) é utilizada como revestimento das placas de microtitulação. Os títulos do anticorpo anticardiolipina IgG parecem ter valor prognóstico e existe dúvida da relevância quando são observados em baixos títulos. A importância do anticorpo anticardiolipina IgM é discutida. Testes para o anticorpo anticardiolipina IgA pode não ter utilidade clínica, exceto quando há historia clínica sugestiva e IgG e IgM negativas?

Devido à natureza heterogênea do anticoagulante lúpico, é necessário mais de um teste diagnóstico. Vários testes laboratoriais foram idealizados para demonstrar o anticoagulante lúpico, sendo que todos detectaram a inibição in vitro das reações de coagulação que são dependentes de fosfolípides. Os testes mais empregados foram: o tempo de tromboplastina parcial ativado (TTPA), tempo do veneno diluído da víbora de Russel (sRVVT) e o tempo de coagulação com caulim (KCT). São testes inespecíficos, porém afetados pela concentração dos fatores da coagulação e pela presença de inibidores que não o anticoagulante lúpico?.

As deficiências dos fatores de coagulação podem ser excluídas por meio da mistura do plasma teste com o plasma normal, levando, assim, à normalização do tempo de coagulação. A dependência de fosfolípides é evidenciada pela redução do tempo de coagulação prolongado, do plasma teste, com a adição de fosfolípides ${ }^{9,35}$ (Quadro 1).

\section{Discussão}

A relação entre os fatores trombofílicos e a infertilidade deve ser considerada pela possibilidade de perda precoce espontânea, abortamento pré-clínico, ocasionada pela alteração da hemostasia de caráter trombofílico no sítio de implantação, que afeta a invasão trofoblástica e a vasculatura placentária ${ }^{44}$.

A formação de trombos nos vasos placentários, tanto arteriais como venosos, impossibilitam a nutrição adequada do embrião, inviabilizando, assim, seu desenvolvimento ${ }^{19}$. As trombo- filias têm sido identificadas com maior frequência em mulheres com falha de implantação submetidas a repetidos ciclos de FIV, quando comparadas com mulheres férteis ${ }^{45}$. A trombofilia também tem sido associada à síndrome de hiperestímulo ovariano (SHO), que ocorre quando há uma resposta ovariana exacerbada após uso de indutores de ovulação, muito utilizada nos procedimentos de FIV $^{43}$.

Alguns estudos sugerem que a síndrome antifosfolípide induz à resistência à proteína $\mathrm{C}$ ativada ${ }^{46} \mathrm{e} / \mathrm{ou}$ interfere na protrombina, proteína $\mathrm{C}$, proteína $\mathrm{S}$, fator tecidual e fator $\mathrm{XI}^{47}$ no sistema da via fator tecidual/inibição do fator tecidual ${ }^{48}$. Outros autores encontraram ainda, em pacientes com síndrome antifosfolípide, anticorpos contra protrombina, proteína $\mathrm{C}$, proteína S, tromboplastina e trombina ${ }^{49,50}$.

Os anticorpos antifosfolípides, anticardiolipina e anticoagulante lúpico são considerados na literatura como fatores etiológicos da falha na implantação e da perda gestacional precoce ${ }^{51}$. Outra proposta é que esses anticorpos são responsáveis pela redução de anexina $\mathrm{V}$, um potente anticoagulante, levando à trombose e, possivelmente, à falha na implantação e à perda gestacional precoce $^{52}$.

A perda fetal é uma manifestação clínica importante da presença dos anticorpos de anticardiolipina por inibirem a secreção da gonadotrofina coriônica, afetando, assim, o desenvolvimento embrionário. Além disso, o anticorpo antifosfolípide interfere em um anticoagulante natural, a proteína placentária anticoagulante (PAP), que se liga com alta afinidade a fosfolipídios aniônicos. O anticorpo antifosfolípide é um inibidor competitivo da PAP. Por essa razão, altos títulos do anticorpo de anticardiolipina podem levar à trombose placentária e à perda fetal ${ }^{13,54}$.

Ademais, os anticorpos antifosfolípides também podem estar associados com o aumento na incidência de complicações obstétricas e pós-natais, incluindo a pré-eclampsia, sofrimento fetal, retardo do crescimento intrauterino, parto prematuro e eventos trombóticos maternos no período pós-parto. A maioria dos casos de perda fetal relacionada com o anticorpo antifosfolípide é precedida de retardo no crescimento intrauterino. A placenta apresenta-se diminuta e, histologicamente, caracteriza-se por uma vasculopatia com infartos difusos ${ }^{55-57}$.

A associação entre a síndrome do aborto recorrente e a síndrome antifosfolípide é evidente, porém pouco elucidada. São necessários mais estudos sobre os mecanismos pelos quais os anticorpos antifosfolípides desencadeiam as perdas fetais. Visando à elucidação desses casos, deve-se também repensar os exames laboratoriais prescritos às pacientes a procura de métodos de fertilização in vitro, pois muitas podem apresentar os anticorpos antifosfolípides e, por isso, não obtêm o sucesso gestacional. E se isso for diagnosticado, podem se submeter às terapias já conhecidas, melhorando, assim, as taxas de sucessos na FIV. 


\section{Referências}

1. Glueck CJ, Awadalla SG, Phillips H, Cameron D, Wang P, Fontaine RN. Polycistic ovary syndrome, infertility, familial thrombophilia, familial hypofibrinolysis, recurrent loss of in vitro fertilized embryos, and miscarriage. Fertil Steril. 2000;74(2):394-7.

2. Grandone E, Colaizzo D, Lo Bue A, Checola MG, Cittadini E, Margaglione M. Inherited thrombophilia and in vitro fertilization implantation failure. Fertil Steril. 2001;76(1):201-2.

3. Torry DS, Leavenworth J, Chang M, Maheshwari V, Groesch K, Ball ER et al. Angiogenesis in implantation. J Assist Reprod Genet. 2007;24(7):303-15.

4. Brenner B. Haemostatic changes in pregnancy. Tromb Res. 2004;114/56):409-14.

5. Lockwood CJ, Nemerson Y, Guller S, Krikun G, Alvarez M, Hausknecht V et al. Progestational regulation of human endometrial stromal cell tissue factor expression during decidualization. J Clin Endocrinol Metab. 1993;76(1):231-6.

6. Runic R, Schatz F, Krey L, Demopoulos R, Thung S, Wan L et al. Alterations in endometrial stromal cells tissue factor protein and messenger ribonucleic acid expression in patients experiencing abnormal uterine bleeding while using Norplant-2 contraception. J Clin Endocrinol Metab. 1997;82(6):1983-8.

7. Lockwood CJ, Krikun G, Papp C, Toth-Pal E, Markiewicz L, Wang EY et al. The role of progestationally regulated stromal cell tissue factor and type1 plasminogen activator inhibitor (PAl-1) in endometrial hemostasis and menstruation. Ann NY Acad Sci. 1994;734:57-79.

8. Nemerson Y. Tissue factor and hemostasis. Blood. 1988;71(1):1-8.

9. Lorenzi TF. Manual de Hematologia: propedêutica e clínica. $4^{\mathrm{a}}$ ed. Rio de Janeiro: Guanabara Koogan; 2006.

10. Drake TA, Morrissey JH, Edgington TS. Selective cellular expression of tissue factor in human tissues. Implications for disorders of hemostasis and thrombosis. Am J Pathol. 1989;134(5):1087-97.

11. Guha A, Bach R, Konigsberg W, Nemerson Y. Affinity purification of human tissue factor: interaction of factor VII and tissue factor in detergent micelles. Proc Natl Acad Sci USA. 1986;83(2):299-302.

12. Bach RR. Initiation of coagulation by tissue factor. CRC Critc Rev Biochem. 1988;23(4):339-68.

13. Moore KL, Persaud TVN. Embriologia Clínica. $7^{a}$ ed. Rio de Janeiro: Elsevier; 2004.

14. Sadler TW. Embriologia Médica. ga ed. Rio de Janeiro: Guanabara Koogan; 2005.

15. Lockwood CJ, Krikun G, Caze R, Rahman M, Buchwalder LF, Schatz F. Decidual cell-expressed tissue factor in human pregnancy and its involvement in hemostasis and preeclampsia-related angiogenesis. Ann N Y Acad Sci. 2008;1127:67-72.

16. Krabbendam I, Franx A, Bots ML, Fijnheer R, Bruinse HW. Thrombophilias and recurrent pregnancy loss: a critical appraisal of the literature. Eur $\mathrm{J}$ Obstet Gynecol Reprod Biol. 2005;118(2):143-53.
17. Dusse LM, Carvalho MG, Cooper AJ, Lwaleed BA. Tissue factor and tissue factor pathway inhibitor: a potencial role in pregnancy and obstetric vascular complications? Clin Chim Acta. 2006;372(1-2):43-6.

18. Bick RL, Hoppensteadt D. Recurrent miscarriage syndrome and infertility due to blood coagulation protein/platelet defects: a review and update. Clin Appl Tromb Hemost. 2005;11(1):1-13.

19. Bick RL. Recurrent miscarriage syndrome and infertility caused by blood coagulation protein or platelet defects. Hematol Oncol Clin North Am. 2000;14(5):1117-31.

20. Inbal A, Muszbeck L. Coagulation factor deficiencies and pregnancy loss. Semin Tromb Hemost. 2003;29(2):171-4.

21. Kumar M, Mehta P. Congenital coagulophaties and pregnancy: report of four pregnancies in a factor X-deficient woman. Am J Hematol. 1994;46(3): 241-4.

22. Valnícek S, Vacl J, Mrázová M, Soska J, Dvorák R, Bílá K. Hemotherapeutic safeguarding of induced abortion in inborn proconvertin insufficiency (hemagglutination factor VII) using exchange plasmapheresis. Zentralbl Gynakol. 1972;94(29):931-5.

23. Nelson DB, Ness RB, Grisso JA, Cushman M. 2001. Influence of hemostatic factors on spontaneous abortion. Am J Perinatol. 18(4):195201.

24. Slunský R. Personal experiences with the antifibrinolytic PAMBA in obstetrics and gynecology. Zentralbl Gynakol. 1970;92(12):364-7.

25. Owen CA Jr, Henriksen RA, McDuffie FC, Mann KG. Prothrombin Quick. A newly identified dysprothrombinemia. Mayo Clin Proc. 1978;53(1): 29-33.

26. Vora S, Shetty $S$, Ghosh K. Coagulation factor deficiency as a cause of recurrent fetal loss: a red herring! Blood Coagul Fibrinolysis. 2007;18(6): $571-4$

27. Evron S, Anteby SO, Brzezinsky A, Samueloff A, Eldor A. Congenital afibrinogenemia and recurrent early abortion: a case report. Eur J Obstet Gynecol Reprod Biol. 1985;19(5):307-11.

28. Bick RL, Baker WF. Antiphospholipid syndrome and thrombosis. Semin Thromb Hemost. 1999;25(3):333-50.

29. Schved JF, Gris JC, Neveu S, Dupaigne D, Mares P. Factor XII congenital deficiency and early spontaneous abortion. Fertil Steril. 1989;52(2):335-6.

30. Klein M, Rosen A, Kyrle P, Beck A. Obstetrical management of dysfibrinogenemia with increased thrombophilia. Geburtshilfe Frauenheilkd. 1992;52(7):442-4.

31. Barkagan ZS, Belykh SI. Protein C deficiency and the multi-thrombotic syndrome associated with pregnancy an abortion. Gematol Transfuziol. 1992;37(9-10):35-7.

32. Hellgren M. Tengborn L, Abildgaard U. Pregnancy in women with congenital antithrombin III deficiency: experience of treatment with heparin and antithrombin. Ginecol Obstet Invest. 1982;14(2):127-41. 
33. Simioni P, Lazzaro AR, Coser E, Salmistraro G, Girolami A. Hereditary heparin cofactor II deficiency and thrombosis: report of six patients belonging to two separate kindreds. Blood Coagul Fibrinolysis. 1990;1(45):351-6.

34. Bick RL. Antiphospholipid syndrome in pregnancy. Hematol Oncol Clin North Am. 2008;22(1):107-20.

35. Santamaria JR, Badziak D, Barros MF, Mandelli FL, Cavalin LC, Sato MS Síndrome antifosfolípide. An Bras Dermatol. 2005;80(3):225-39.

36. Buckingham KL, Chamley LW. A critical assessment of the role of antiphospholipid antibodies in infertility. J Reprod Immunol. 2009;80(1-2):132-45.

37. Wassermann A, Neisser A, Bruck C. Eine serodiagnostiche reaktion bei syfhilis. Deutsche Medicische Wochenschrift. 1906;32:745-6.

38. Pangborn MC. A new serologically active phospholipid from beef heart. Proc Soc Exp Biol Med. 1941;48:484-6.

39. Conley CL, Hartmann RC. A hemorrhagic disorder caused by circulating anticoagulant in patients with disseminated lupus erythematousus. J Clin Invest. 1952;31:621-2.

40. Feinstein DI, Rapaport SI. Acquired inhibitors of blood coagulation. Prog Hemost Thromb. 1972;1:75-95.

41. Bowie EJ, Thompson JH Jr, Pascuzzi CA, Owen CA Jr. Thrombosis in systemic lupus erythematosus despite circulating anticoagulants. J Lab Clin Med. 1963;62:416-30.

42. Hughes GR. The anticardiolipin syndrome. Clin Exp Rheumatol. 1985;3(4):285-6.

43. Soligo AGS, Barini R, Carvalho ECC, Annichino-Bizzacchi J. Prevalência dos fatores trombofílicos em mulheres com infertilidade. Rev Bras Ginecol Obstet. 2007;29(5):235-40.

44. Sarto A, Rocha M, Geller M, Capmany C, Martinez M, Quintans C et al. Tratamiento con enoxaparina adaptado a los programas de fertilidad en mujeres con aborto recurrente y trombofilia. Medicina (Buenos Aires). 2001;61(4):406-12.

45. Azem F, Many A, Ben Ami I, Yovel I, Amit A, Lessing JB et al. Increased rates of thrombophilia in women with repeated IVF failures. Hum Reprod. 2004:19(2):368-70.
46. Aznar J, Villa P, España F, Estellés A, Grancha S, Falcó C. Activated protein $\mathrm{C}$ resistance phenotype in patients with antiphospholipid antibodies. J Lab Clin Med. 1997;130(2):202-8.

47. Schultz DR. Antiphospholipid antibodies: basic immunology and assays. Semin Arthritis Rheum. 1997:26(5):724-39.

48. Amengual 0 , Atsumi T, Khamashta MA, Hughes GR. The role of the tissue factor pathway in the hypercoagulable state in patients with the antiphospholipid syndrome. Thromb Haemost. 1998;79(2):276-81.

49. Martini A, Ravelli A. The clinical significance of antiphospholipid antibodies. Ann Med. 1997;29(2):159-63.

50. Bussen SS, Steck T. Thyroid antibodies and their relation to antithrombin antibodies, anticardiolipin antibodies and lupus anticoagulant in women with recurrent spontaneous abortions (antithyroid, anticardiolipin and antithrombin autoantibodies and lupus anticoagulant in habitual aborters) Eur J Obstet Gynecol Reprod Biol. 1997;74(2):139-43.

51. Qublan HS, Eid SS, Ababneh HA, Amarin ZO, Smadi AZ, Al-Khafaji FF et al. Acquired and inherited thrombophilia: implication in recurrent IVF and embryo transfer failure. Hum Reprod. 2006;21(10):2694-8.

52. Rand $\mathrm{JH}, \mathrm{Wu} \mathrm{XX}$. Antibody-mediated disruption of the annexin-V antithrombotic shield: a new mechanism for thrombosis in the antiphospholipid syndrome. Thromb Haemost. 1999;82(2):649-55.

53. Aron AL, Gharavi AE, Shoenfeld Y. Mechanisms of action of antiphospholipid antibodies in the antiphospholipid syndrome. Int Arch Allergy Immunol. 1995;106(1):8-12.

54. Meroni PL, Del Papa N, Borghi MO. Antiphospholipid and antiendothelial antibodies. Int Arch Allergy Immunol. 1996;111(4):320-5.

55. Harris EN, Khamashta MA, Hughes GRV. Antiphospholipid antibody syndrome. In: Mccarty DJ, Koopman WJ, editors. Arthritis and allied conditions. $13^{\text {th }}$ ed. Philadelphia: Lea \& Febiger; 1997. p. 1201-13.

56. Gharavi AE, Wilson WA. Antiphospholipid antibodies. In: Wallace DJ, Hahn BH, editors. Dubois' lupus erythematosus. $5^{\text {th }}$ ed. Baltimore, MD, USA: Williams \& Wilkens; 1997. p. 471-92.

57. Welsch S, Branch DW. Antiphospholipid syndrome in pregnancy. Obstetric concerns and treatment. Rheum Dis Clin North Am. 1997:23(1):71-84. 